ISSN 0280-5316

ISRN LUTFD2/TFRT--5645--SE

\title{
Robust tuning procedures of dead-time compensating controllers
}

Ari Ingimundarson

Department of Automatic Control Lund Institute of Technology December 2000 


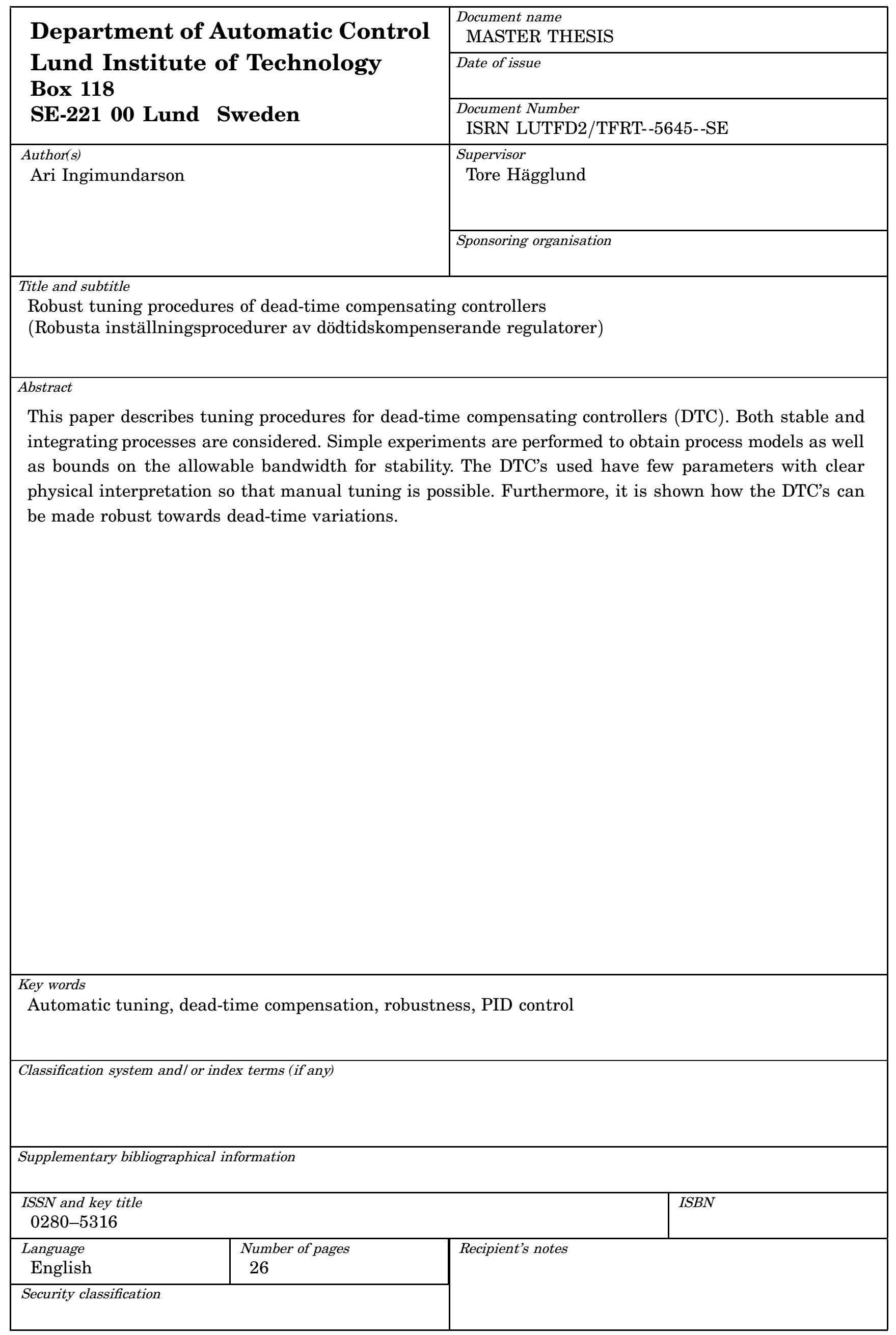

The report may be ordered from the Department of Automatic Control or borrowed through: 


\section{Introduction}

Most control problems in the process industry are solved using PID controllers. There are several reasons for this. One is that the PID controller can be tuned manually by "trial-and-error" procedures, since it only has three adjustable parameters. The possibility to make manual adjustments of the controller parameters is important even when automatic tuning procedures are available.

When there are long dead times in the process, the control performance obtained with a PID controller is, however, limited. For these processes, dead-time compensating controllers (DTC) may improve the performance considerably. These controllers require a process model to provide modelpredictive control. This usually means a significant increase in controller parameters.

The use of DTC's also brings into existence new robustness problems connected to the dead-time. The classical ways to characterize robustness, phase margin and amplitude margin are not sufficient. In this paper, the delay margin which is the greatest variation in dead-time that can occur in the process before the closed-loop system becomes unstable, will be used as well.

The aim of this paper is to show how it is possible with simple experiments to find parameters for the DTC's that give good performance while remaining robust. The experiments are composed of an identification of simple process models and then an experiment to determine an upper limit on closed-loop bandwidth. The latter is performed in open loop while the former is partially performed in closed-loop. As a measure of closed-loop bandwidth, the reciprocal of the time constant of the set point response is used. This can then be related to other measures such as the loop-gain crossover frequency. The DTC's used in this paper have certain PID qualities, i.e. few parameters that can be tuned manually and have good interpretation in terms of classical control theory concepts. It will also be shown how the DTC's can be given a guaranteed delay margin.

For the identification of the simple process models in the DTC's an identification method first presented in [Ingimundarson and Hägglund, 2000a] is used. In this paper only the main equations and results are presented.

The paper is arranged in the following manner. In section 2 the identification method is introduced. In section 3, dead-time compensating controllers are discussed. In section 4 the tuning procedure for stable processes is presented. This is followed by the procedures for integrating processes in section 5. Finally conclusions are drawn in section 6 .

\section{Identification}

The two processes that are identified are the first-order plus dead-time (FOPDT)

$$
P_{n}(s)=\frac{K_{n}}{T_{n} s+1} e^{-L_{n} s}
$$

and the two-parameter model

$$
P_{n}(s)=\frac{K_{n}}{s} e^{-L_{n} s}
$$


These models are frequently used in the process industry and are considered to capture dynamics of real plants sufficiently well for many applications.

The identification method presented in this paper can be divided into two phases. First the average residence time, $T_{a r}=L_{n}+T_{n}$ and the gain $K_{n}$ are estimated with a change in operating levels. This change can be accomplished by a change in set point while operating in closed loop. The approach is based on the method of moments, see [Åström and Hägglund, 1995] for a general input signal applied to a linear system initially at rest. Secondly the apparent time constant $T_{n}$ is determined with an open loop experiment where the input signal is a step or a ramp. In the case of the two-parameter model given by Eq. (2) only the first part of the experiment is necessary.

\subsection{The method of moments}

The method of moments can be explained with the following equations. For a general transfer function $G(s)$ an arbitrary input signal $U(s)$ results in an output signal given by

$$
Y(s)=G(s) U(s)
$$

By derivating $Y(s)$ with regard to $s$ one gets

$$
Y^{\prime}(s)=G^{\prime}(s) U(s)+G(s) U^{\prime}(s)
$$

The transfer function $\mathrm{G}(\mathrm{s})$ and its derivative can be evaluated at an arbitrary point $\alpha$ by calculating

$$
\begin{aligned}
& Y^{(n)}(\alpha)=(-1)^{n} \int_{0}^{\infty} t^{n} e^{-\alpha t} y(t) d t \\
& U^{(n)}(\alpha)=(-1)^{n} \int_{0}^{\infty} t^{n} e^{-\alpha t} u(t) d t
\end{aligned}
$$

and solving Eqs.(3) and (4). Notice that if $\alpha=0$ it is necessary for the signals considered to go to zero as time goes to infinity. Otherwise the integrals will not converge. Typically an input signal of the sort $\bar{u}(t)=$ $u(\infty)-u(t)$ is selected. $u(\infty)$ is the value of the input signal after steady state has been reached again. The corresponding output signal is then $\bar{y}(t)=y(\infty)-y(t)$. Then it is only necessary to integrate for a finite time interval. This interval is denoted $\left[t_{b}, t_{f}\right]$ in the following.

In the case of the FOPDT model, (Eq. 1) it is easy to get the following expression

$$
\frac{P_{n}^{\prime}(s)}{P_{n}(s)}=-L_{n}-\frac{T_{n}}{1+T_{n} s}
$$

By evaluating the transfer function at $\alpha=0, T_{a r}$ can be written as

$$
T_{a r}=-\frac{P_{n}^{\prime}(0)}{P_{n}(0)}
$$

To evaluate $P_{n}^{\prime}(0)$ it would be necessary to calculate the first moment of $\bar{y}(t)$ and $\bar{u}(t)$ for signals for which the integrals converge. These integrals have bad noise properties because of the factor $t$. Values at the end of the 
experiment have much higher weight than the ones in the beginning of the experiment. Therefore it is beneficial to consider the artificial signals

$$
\begin{aligned}
& y_{d}(t)=\frac{\mathrm{d}}{\mathrm{d} t} \bar{y}(t) \\
& u_{d}(t)=\frac{\mathrm{d}}{\mathrm{d} t} \bar{y}(t)
\end{aligned}
$$

The novelty of the method is the use of these signals. Denoting the $n$-th moment of $y_{d}$ and $u_{d}$ with $y_{n}$ and $u_{n}$ respectively it is possible to evaluate $P_{n}^{\prime}(0)$ as

$$
P_{n}^{\prime}(0)=\frac{y_{1}-P_{n}(0) u_{1}}{u_{0}}
$$

Evaluating the moment integrals $y_{0}$ and $y_{1}$ gives

$$
\begin{aligned}
y_{0} & =\int_{0}^{\infty} y_{d}(t) d t=[\bar{y}(t)]_{0}^{\infty} \\
& =-\bar{y}(0) \\
y_{1} & =-\int_{0}^{\infty} t y_{d}(t) d t \\
& =-[t \bar{y}(t)]_{0}^{\infty}+\int_{0}^{\infty} \bar{y}(t) d t \\
& =\int_{0}^{\infty} \bar{y}(t) d t
\end{aligned}
$$

By evaluating Eq. (5) with moment equations Eqs. (7) and (8) the following expression is derived

$$
\begin{aligned}
T_{a r} & =-\frac{P_{n}^{\prime}(0)}{P_{n}(0)}=\frac{-y_{1}+P_{n}(0) u_{1}}{u_{0} P_{n}(0)} \\
& =\int_{t_{b}}^{t_{f}} \frac{\bar{y}(t)}{\bar{y}(0)} d t-\int_{t_{b}}^{t_{f}} \frac{\bar{u}(t)}{\bar{u}(0)} d t
\end{aligned}
$$

This can be rewritten as

$$
T_{a r}=\int_{t_{b}}^{t_{f}} \frac{u(t)-u\left(t_{b}\right)}{u\left(t_{f}\right)-u\left(t_{b}\right)} d t-\int_{t_{b}}^{t_{f}} \frac{y(t)-y\left(t_{b}\right)}{y\left(t_{f}\right)-y\left(t_{b}\right)} d t
$$

The limits of the integrals have been changed to $t_{b}$ and $t_{f}$. Eq. (10) has a nice graphical explanation. The functions under the integrals have beginning value equal to zero and final values equal to one. $T_{a r}$ is simply the area between the two signals when they go from 0 to 1 . This is shown in Fig. 1.

The method presented requires a change in the process levels. If this is accomplished by changing the set-point in closed-loop a stable controller is assumed to be present. The form of this change is not important but a step or a ramp would be easiest to implement in practice. Since the identification is based on integrals of the input and output signals it is preferable that the experiment is as short as possible in the presence of measurement noise. 


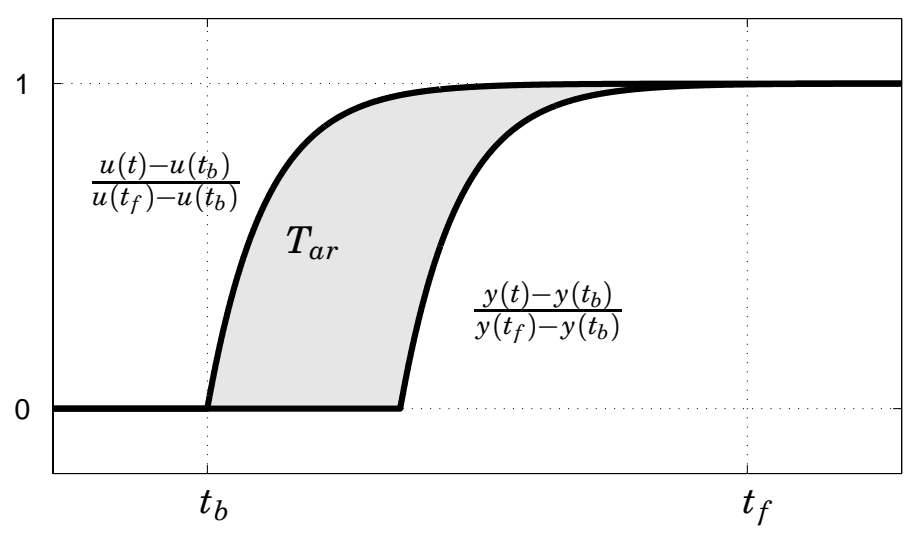

Figure 1 Graphical explanation for Eq. (10)

If an integrator is present in the process the method presented can be used in the same way by considering the integral of the process input signal

$$
u_{i}(t)=\int_{t_{b}}^{t} u(t) d t
$$

and replacing $u_{i}(t)$ with the input signal in the previous paragraphs. Deadtime $L_{n}$ in Eq. (2) is then given by $T_{a r}$

Process gain $K_{n}$ is trivially estimated as the ratio between input and output signal change, $K_{n}=\bar{y}(0) / \bar{u}(0)$.

\subsection{Open loop experiment to identify $T_{n}$}

If a step or a ramp is applied to the FOPDT model analytical expressions for the output signal are easily obtained. By integrating these expressions from 0 to $T_{a r}$ it is possible to get expressions with only $T_{n}$ as the unknown. In Eqs. (11) and (12) $T_{n}$ is given for a step or ramp input signal.

$$
\begin{aligned}
\text { Step : } & T_{n}=\frac{A e^{1}}{h K_{n}} \\
\text { Ramp : } & T_{n}=\sqrt{\frac{A}{h K_{n}\left(1 / 2-e^{-1}\right)}}
\end{aligned}
$$

Parameter $A$ is the integral of $y(t)$ from 0 to $T_{a r}$. The parameter $h$ is the amplitude for a step signal or the rate for a ramp signal. The length of the open loop experiment is always $T_{a r}$.

Notice that Eq. (12) can be used for a FOPDT model with integrator as well. Sending a step of height $h$ to an integrating process is the same as sending a ramp with rate $h$ to the process without an integrator. In both cases Eq. (12) can be used to find $T_{n}$. The problem with the open-loop ramp experiment is that if the dead-time $L_{n}$ is sufficiently larger than time constant $T_{n}$, the final value of output signal $y(t)$ will be quite large if one selects a large $h$. But it is necessary for good estimation of the integral $A$ to have a large $h$. This limits the use of this extension to cases where there is little measurement noise. 

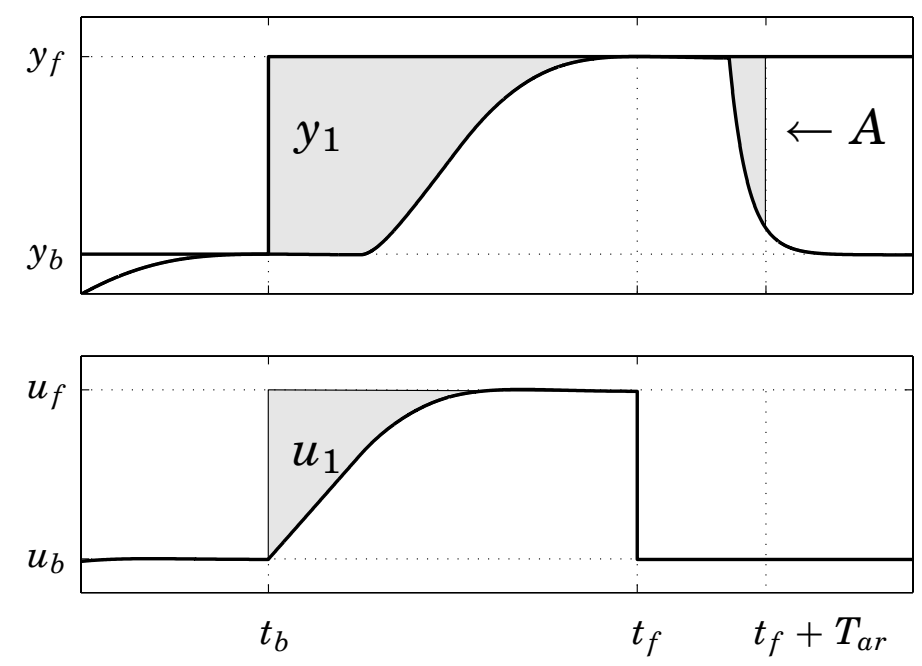

Figure 2 Identification experiment for $G(s)=\frac{1}{s+1} e^{-5 s}$

\subsection{Identification procedure}

The basic steps in the identification procedure can now be presented. In Fig. 2. the input and output signals are shown for a specific FOPDT process. For simplicity the change in set-point for the closed loop experiment is a step. The open loop experiment is also a step.

1. Control the process to a steady state initial level $y_{b}$. Record the signal levels $u_{b}$ and $y_{b}$.

2. Apply a step in the reference signal $y_{s p}(t)$ at time $t_{b}$

3. Integrate $y(t)$ and $u(t)$ until process reaches steady state again. This occurs at time $t_{f}$. Again record the signal levels $u_{f}$ and $y_{f}$.

4. Determine process gain $K_{n}$ by observing the signal levels and $T_{a r}$ from Eq. (10).

5. Apply a step in open loop and integrate the area $A$ using the estimate of $T_{a r}$ obtained from previous step.

6. Estimate time constant $T_{n}$ from Eq. (11) and dead time by $L_{n}=$ $T_{a r}-T_{n}$.

The method requires a decision criterion on when steady state has been achieved. This occurs at times $t_{b}$ and $t_{f}$. If integral action is present in the controller the steady state error should be zero. This reduces the task to determining when $y(t)$ is the same as the set-point $y_{s p}(t)$.

In the presence of noise the steady state values $u_{b}, u_{f}, y_{b}$ and $y_{f}$ can be determined by averaging the signals over a period of time.

In the presence of measurement noise the numerical evaluation of the integrals might have large variance. Less variance would be assured by proper noise filtering. The noise filter would then of course be considered as part of the controlled process. 


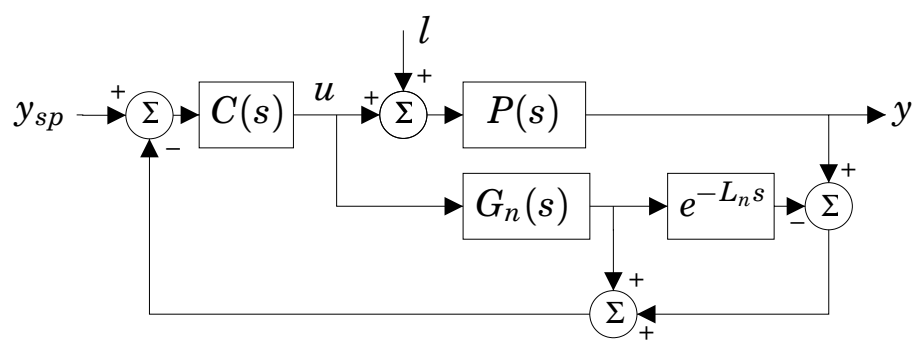

Figure 3 Block diagram of a Smith predictor.

\section{Dead-time compensation}

The most common dead-time compensating controller is the Smith Predictor (SP) [Smith, 1957]. The structure of this controller is shown in Fig. 3. The controller output $u$ is fed through a model of the process and through the same model without dead time. In this way, the controller acts, in the ideal situation of perfect modeling, on a simulated process which behaves as if there was no dead time in the process.

The real process is assumed to be linear time invariant and is denoted as $P(s)$. The model is denoted $P_{n}(s)=G_{n}(s) e^{-L_{n} s} \cdot G_{n}(s)$ is delay free.

A SP which uses the simple FOPDT model given by Eq. (1) combined with a PI controller

$$
C(s)=K\left(1+\frac{1}{s T_{i}}\right)
$$

requires five parameters to be determined, namely $K, T_{i}, K_{n}, T_{n}$, and $L_{n}$. These parameters may be obtained from a systematic process identification experiment. However, it is practically impossible to tune this controller manually by trial and error procedures. Therefore, replacing a PID controller with a standard SP gives a drastic increase in operational complexity. This increase is present in both the commissioning and maintenance of dead-time compensating controllers.

A common way to deal with this complexity is to automize the tuning procedure. Automatic tuning of DTC's has received some attention in the literature, some references are [Palmor and Blau, 1994], [Lee et al., 1995] and [Vrancić et al., 1999]. But even when automatic tuning procedures are available simpler structures are advantageous since it provides a possibility for the user to make the last final adjustments manually or manually re-tune the controller later.

A few papers have been written that emphasize the importance of less complex DTC's. In the stable case, [Hägglund, 1996] is one example and in the case of integrating processes [Matausek and Micic, 1999] have addressed the problem.

The bandwidth of DTC's is usually related to the model parameters which are assumed to be available when the DTC's are initially tuned. In [Palmor and Blau, 1994], the closed-loop time constant was set proportional to the apparent dead-time of the process. In [Hägglund, 1996] it was related to the open-loop apparent time constant. In [Normey-Rico et al., 1997] the closed-loop bandwidth was related to both of these.

In the case of integrating processes, it has been more common that the initial bandwidth is supposed to be manually tuned. Guidelines are 
given from where a starting point can be obtained. In [Normey-Rico and Camacho, 1999] the closed-loop time constant was related to an assumed dead-time error between the model and process. In [Matausek and Micic, 1999] it was suggested that the closed-loop time constant should be set equal to the apparent time constant of the dynamics additional to the integrator.

In this paper a new approach is taken to determine the closed-loop timeconstant in the initial tuning. Given the model parameters it is possible to calculate the uncertainty norm boundary of the DTC's. The uncertainty norm boundary tells how much the real process can deviate from the model at each frequency without the closed loop system becoming unstable. Then it is shown how it is possible with simple experiments to obtain frequency dependent inequalities bounding the model uncertainty. A lower bound on the closed-loop time constant is then found by making sure the model uncertainty found is always less than the uncertainty norm boundary of the DTC's. The goal is that this initial tuning is, when the model is close to the process, less conservative but robustly stable.

It was mentioned in the introduction that classical measures of robustness such as gain and phase margin are not sufficient when dealing with dead-time systems. This is discussed in [Palmor, 1980]. In addition to these classical ones it is proposed that a third one is used, namely the delay margin. The delay margin of a closed-loop system can be defined in the following way (modifying slightly the definition in [Landau et al., 1995]). If the Nyquist curve intersects the unit circle at frequencies $\omega_{i}$ with the corresponding phase margins $\Phi_{i}$ then the delay margin can be defined as

$$
D_{M}=\min _{i} \frac{\Phi_{i}}{\omega_{i}}
$$

Most of the tuning rules for DTC's presented in the literature provide a certain delay margin. The initial tuning, which results from the procedures in this paper, can have an arbitrary small delay margin if the model describes the process well. Therefore it is shown how the DTC's can be re-tuned with a guaranteed delay margin. This can have a practical value when it is known how much the dead-time might vary around the operating point. Finally it is also shown what delay margin can be expected from the initial tuning in the nominal case, i.e. when the model and process are equal.

\section{Stable Case}

In [Hägglund, 1996], a dead-time compensating controller with only three adjustable parameters was presented. The controller can be viewed as a PI controller with model-based prediction. The abbreviation PPI stands for "Predictive PI". The controller can be tuned manually in the same way as a PID controller.

The structure of the PPI controller is the same as for the Smith predictor, with the FOPDT model (1) combined with the PI controller (13). The only difference is the parameterization. The five adjustable parameters are reduced to three by introducing constraints between the controller 


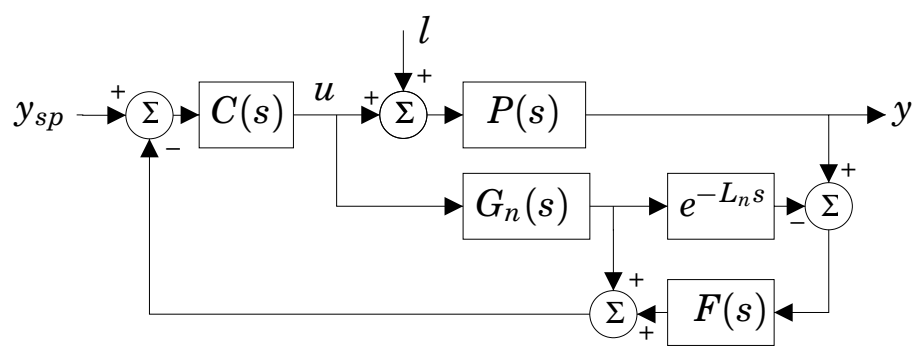

Figure 4 The FPPI

parameters and the model. These constraints are

$$
\begin{aligned}
T_{n} & =T_{i} \\
K_{n} & =\kappa / K
\end{aligned}
$$

$\kappa$ is a constant to be determined later. The identification method presented before only provides a good approximation of the real process at low frequencies. Robustness problems for PPI can occur because of model error at high frequencies. In [Normey-Rico et al., 1997] a filter was proposed to provide robustness towards high frequency model errors. The resulting controller was abbreviated FPPI. The proposed controller structure can be seen in Fig. 4. The filter $F(s)$ is typically a one parameter low-pass filter and it is shown later that it is sufficient for the purpose of this paper to assume it is of first order

$$
F(s)=\frac{1}{T_{f} s+1}
$$

In the nominal case, that is when the model describes the process perfectly, $P=P_{n}$, the controller parameterization given by Eq. (14) results in a closed loop set point response

$$
\frac{Y(s)}{Y_{s p}(s)}=\frac{1}{T_{r} s+1} e^{-L_{n} s}
$$

where $T_{r}=T_{n} / \kappa$. This is a familiar result regarding Smith-predictors. The time constant of the closed loop system can be reduced by increasing $\kappa$. Notice that $\kappa=1$ places the closed-loop pole in the same place as the open loop one. A proper selection of $T_{r}$ will be the main subject of the next subsection. It is shown how an lower limit on $T_{r}$ can be obtained by performing a simple open-loop step experiment.

\subsection{Selection of $T_{r}$}

The closed loop characteristic equation for the FPPI is

$$
1+\frac{C}{1+C G_{n}} F\left(P-P_{n}\right)=0
$$

Denoting the difference between model and process as $\delta P=P-P_{n}$ the maximum value of $|\delta P|$ allowable while maintaining closed loop stability, 
or the uncertainty norm boundary can be obtained from Eq. (16) by solving for $\delta P$

$$
|\Delta P|_{\mathrm{FPPI}}=K_{n}\left|\frac{\left(i \omega T_{r}+1\right)\left(i \omega T_{f}+1\right)}{\left(i \omega T_{n}+1\right)}\right|
$$

Notice that if an inequality of type

$$
|\delta P| \leq A \omega
$$

is available the system can be made stable by chosing appropriately $T_{r}$ and $T_{f}$. This follows from the fact that the degree of the denominator polynomial is one higher than the numerator polynomial. The condition for stability would then be

$$
|\delta P| \leq A \omega<|\Delta P|_{\text {FPPI }} \quad \forall \omega
$$

An uncertainty bound of type (18) can be obtained with a simple openloop step experiment. The step response of the real system is denoted by $y(t)$. After an identification experiment the FOPDT model response is available and given by

$$
y_{n}(t)= \begin{cases}0 & \text { for } t<L_{n} \\ K_{n}\left(1-e^{\left.-\left(t-L_{n}\right) / T_{n}\right)}\right. & \text { for } t>L_{n}\end{cases}
$$

Denoting the difference between the two responses $f(t)=y(t)-y_{n}(t)$, the following expression is the definition of the Laplace transform

$$
\frac{P(s)-P_{n}(s)}{s}=\int_{0}^{\infty} e^{-s t} f(t) d t
$$

Putting $s=i \omega$ the following equation is obtained

$$
\begin{aligned}
\left|\frac{P(i \omega)-P_{n}(i \omega)}{i \omega}\right| & =\left|\int_{0}^{\infty} e^{-i \omega t} f(t) d t\right| \\
& \leq \int_{0}^{\infty}|f(t)| d t \\
& =A
\end{aligned}
$$

Note that the error is weighted with one over $\omega$. At stationarity therefore there can be no error. Therefore $P_{n}(s)$ and $P(s)$ have to have the same steady state gain $K_{n}$.

If a time constant $\bar{T}_{f}=A / K_{n}$ is defined the relevant areas can be graphically displayed on a normalized step response. This is shown in Fig. 5.

Inequality (19) can now be restated the following way

$$
\left\|\frac{\bar{T}_{f} \omega\left(i T_{n} \omega+1\right)}{\left(i T_{r} \omega+1\right)\left(i T_{f} \omega+1\right)}\right\|_{\infty}<1
$$

Notice that $\bar{T}_{f}$ and $T_{n}$ are assumed to be known while $T_{r}$ and $T_{f}$ are design parameters. The latter two should be chosen to minimize some performance criteria while fulfilling the above inequality. For a fast set point 


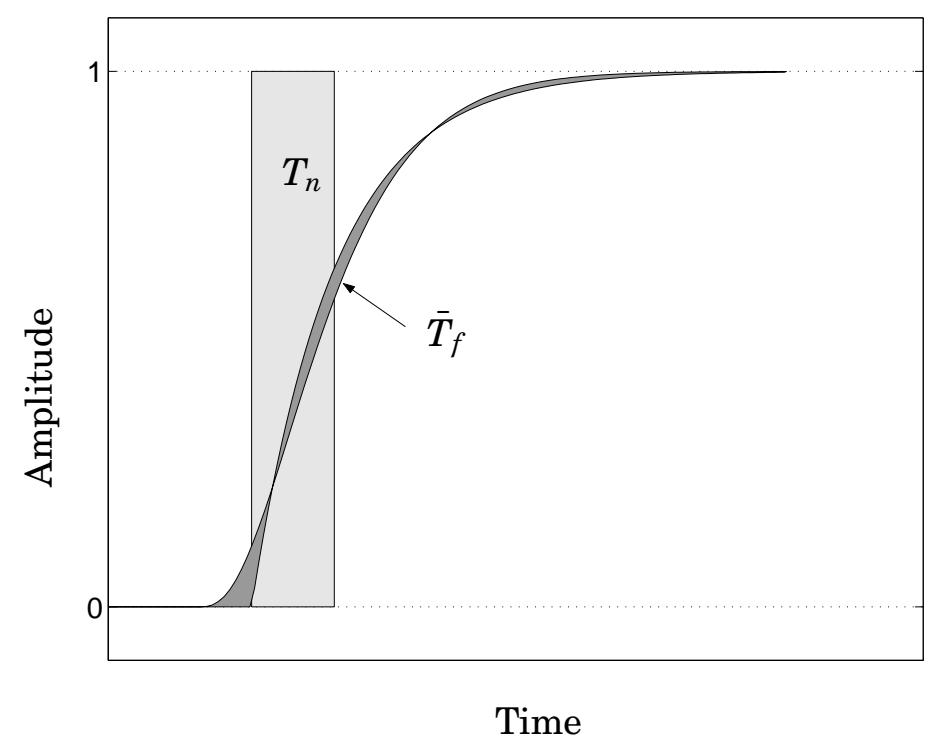

Figure 5 Step responses $y(t)$ and $y_{n}(t)$ normalized to 1 .

response, $T_{r}$ could be chosen small while $T_{f}$ would be used to fulfill the above inequality. Commonly in process control, regulatory performance is considered more important. The performance criteria recommended here is to minimize the integrated error when load disturbance $l$ is a unit step. Using the final value theorem the following expression is obtained.

$$
\int_{0}^{\infty} e(t) d t=\left(T_{r}+T_{f}+L_{n}\right) K_{n}
$$

The design problem can be set up as a minimization problem where Eq. (24) is the cost function and Eq. (23) is the constraint. This problem can be further simplified. Notice that $T_{r}$ and $T_{f}$ enter the cost function and the constraint the same way. Using this fact it is possible to obtain necessary conditions that show that at the optimum, $T_{r}$ is equal to $T_{f}$. Still the analytic solution to this problem quickly becomes rather involved. A necessary condition is that the direct term in the transfer function is less than 1 . This gives the following condition

$$
T_{r}>\sqrt{T_{n} \bar{T}_{f}}
$$

A further simplification of the problem can be obtained by normalizing the frequency in inequality (23) with $T_{r}$. If the following quantities are defined

$$
\bar{\omega}=T_{r} \omega \quad \gamma=\bar{T}_{f} / T_{r}
$$

inequality (23) can be written as

$$
\left\|\frac{\gamma \bar{\omega}(i \kappa \bar{\omega}+1)}{(i \bar{\omega}+1)^{2}}\right\|_{\infty}<1
$$

$\kappa$ was defined following Eq. (15). Using a bisection algorithm, an upper limit on $\kappa$ for which inequality (26) holds, was calculated as a function of 


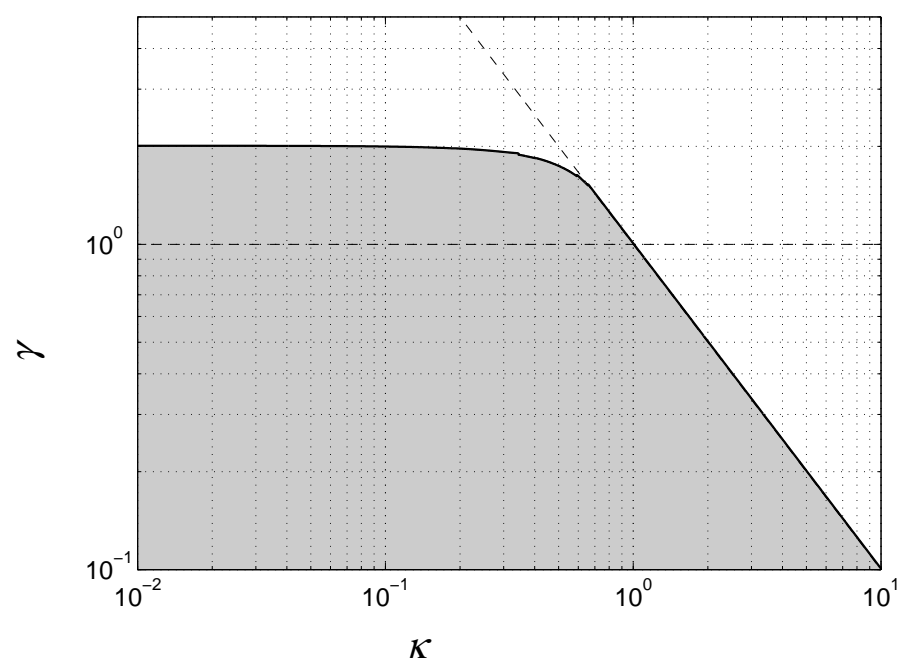

Figure $6 \kappa$ and $\gamma$ within the shaded area fulfill inequality (26). The edge of the shaded area corresponds to the equality. Also shown are design lines $\kappa=1 / \gamma$ and $\gamma=1$

$\gamma$. This is shown in Fig. 6. Any pair of $\kappa$ and $\gamma$ that lies within the shades area fulfills inequality (26). Using the above figure the following design rules are proposed

$$
\gamma \kappa \leq 1 \quad \gamma \leq 1
$$

In terms of the time constants this becomes

$$
\begin{aligned}
& T_{r} \geq \sqrt{\bar{T}_{f} T_{n}} \\
& T_{r} \geq \bar{T}_{f}
\end{aligned}
$$

Remark The use of an equality in Eq. (28) requires justification. Inequality (22) allows infinite error when $\omega \rightarrow \infty$. Most normal processes are on the other hand of low-pass character. This means the inequality could be replaces with a strict inequality at high frequencies. When $\kappa \gamma=1$ the supremum of the norm in Eq. (26) is achieved when $\omega \rightarrow \infty$. So using additional information about inequality (22), Eq. (28) can be justified.

\subsection{Sensitivity to dead-time errors}

Robustness of DTC's has been analyzed by many authors. Some references are [Morari and Zafiriou, 1989], [Palmor, 1980] and [Lee et al., 1996]. Usually most attention is devoted to analyzing the sensitivity towards errors in the dead-time. The reason for this is that it is often towards these errors dead-time compensators are most sensitive.

With this in mind it is important to give easy ways to reduce the deadtime sensitivity and be able to set a desired delay margin without limiting the bandwidth, i.e. sacrificing performance, to much. If the dead-time is expected to vary an amount $\Delta L$ the increase in integral $A$ in Eq. (22) is at most $K_{n} \Delta L$. So an arbitrary delay margin can be set by adding the expected dead-time variation to $\bar{T}_{f}$ and recalculating $T_{r}$ with Eq. (28).

Using the result of [Palmor, 1980] one can see that with the tuning presented in this section, the resulting DTC has phase margin $60^{\circ}$ and 
gain margin equal to 2 in the nominal case. The way to see this is to note that the loop-gain is

$$
L(i \omega)=\frac{e^{-i L_{n} \omega}}{\left(i T_{r} \omega+1\right)^{2}-e^{-i L_{n} \omega}}
$$

which can be written as

$$
L(i \omega)=\frac{N(i \omega)}{1-N(i \omega)}
$$

where $N(i \omega)$ is a frequency response with amplitude less than 1 for all $\omega$.

Further insight can be obtained into the relation between the delay margin and closed-loop time constant $T_{r}$ by normalizing the variables of the loop-gain with $L_{n}$. This is the same approach as was taken in [Palmor and Blau, 1994]. If the process is equal to the model except for an error in the dead-time, $L=L_{n}+\delta L$, the loop-gain becomes

$$
L(s)=\frac{e^{-L s}}{\left(T_{r} s+1\right)^{2}-e^{-L_{n} s}}
$$

If the variables are normalized with $L_{n}$ the following dimensionless variables are obtained.

$$
\delta_{L}=\delta L / L_{n} \quad \bar{\omega}=\omega L_{n} \quad \bar{T}_{r}=T_{r} / L_{n}
$$

The normalized loop-gain is then

$$
\bar{L}(i \bar{\omega})=\frac{e^{-i \bar{\omega}\left(1+\delta_{L}\right)}}{\left(i \bar{T}_{r} \bar{\omega}+1\right)^{2}-e^{i \bar{\omega}}}
$$

The relationship between the two loop-gains is $\bar{L}\left(i \omega L_{n}\right)=L(i \omega)$. By using the Nyquist criterion it is possible to calculate a lower limit on $\bar{T}_{r}$ for which the closed-loop is stable, as a function of $\delta_{L}$. This is shown in Fig. 7. The figure shows that if $\bar{T}_{r} \geq 0.22, \delta_{L}$ can vary from -1 to 1 without the closedloop system becoming unstable. It also shows that very aggressive tuning, $\bar{T}_{r}=0.01$ would give a relative delay margin from -0.2 to 0.15 .

It is of interest to see what time constant $\bar{T}_{r}$ is obtained by specifying a desired delay margin, $\Delta L$ and calculating $\bar{T}_{r}$ from Eq. (28). Dividing by $L$

$$
\bar{T}_{r}=\sqrt{\frac{\Delta L}{L} \frac{T_{n}}{L}}
$$

In Fig. $7 \bar{T}_{r}$ is plotted as a function of $\Delta L / L$ for different values of $T_{n} / L$. Also shown is $\bar{T}_{r}$ calculated with Eq. (29). The recommended $\bar{T}_{r}$ is well above the stability limit so a certain degree of robust performance should be assured. Notice that when $T_{n}$ is close to $L_{n}$ the tuning can be quit conservative. Increasing dead-time results in lower delay-margins.

The lower limit on $\bar{T}_{r}$ in Fig. 7 is calculated for the nominal case. Experience from simulations indicate that this lower limit is not a bad approximation for other processes, specially if $\bar{T}_{f} / T_{n}$ is little. Notice that the method of setting a desired delay margin by adding $\Delta L$ to $\bar{T}_{f}$ is valid for any process. 


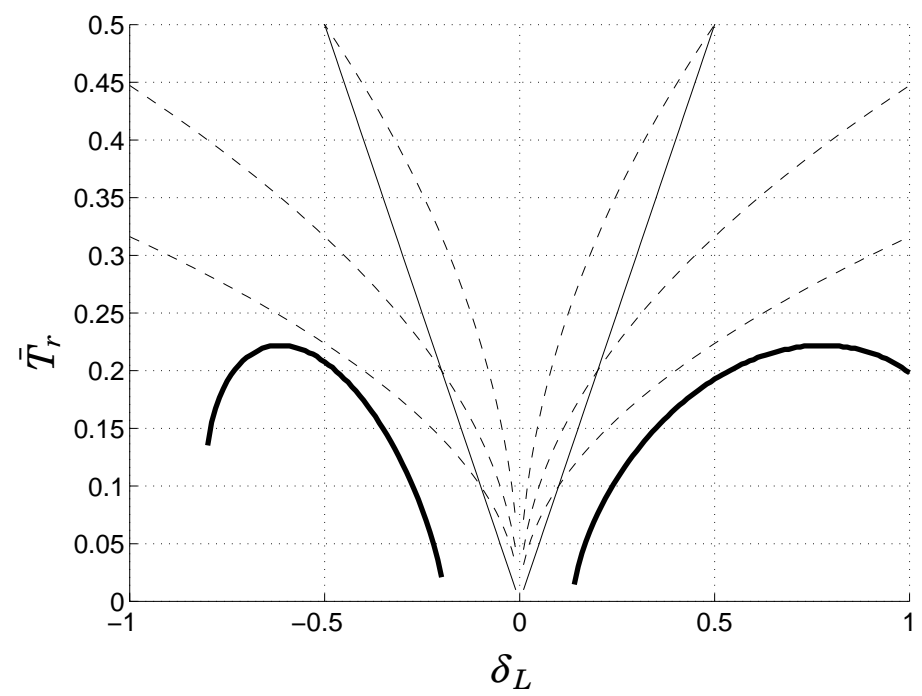

Figure 7 Thick line is the lower bound on $\bar{T}_{r}$ for a given error $\delta_{L}$. The dashed lines are the design recommendation for $\bar{T}_{r}$ for $T_{n} / L_{n}$ equal to $0.5,0.2$ and 0.1 counting from above. Finally the thin solid line is the design rule given by Eq. (29).

\subsection{Practical issues}

Some comments are in order relating to the practical use of the FPPI and specially the selection of $T_{r}$.

- If the model is close to the real process, $\bar{T}_{f}$ will be small resulting in a small $T_{r}$. Other limiting factors such as actuator saturation will then come into the picture.

- The performance of the FPPI is always limited by the dead-time of the process. Therefore tuning aggressively by selecting a small $T_{r}$ might only give marginal improvements. In the nominal case this is apparent from Eq. (24). This fact can be used to motivate tuning rules. One can decide how large portion of the integrated error comes from $L_{n}$, which one can do nothing about. From this and equation relating $L_{n}$ and $T_{r}$ can easily be obtained.

- Looking at Fig 7 while keeping Eq. (24) in mind, the tradeoff between performance and robustness is apparent. Robustness in terms of delay margin costs in terms of increased $T_{r}$.

- The value of controller parameter $T_{f}$ should be equal to $T_{n} / \kappa=T_{i} / \kappa$. The FPPI has then 3 parameters to tune, namely $K, T_{i}$ and $L_{n}$. After the open-loop experiment where $\bar{T}_{f}$ is obtained and a delay margin $\Delta L$ has been decided upon, $\kappa$ can be calculated as

$$
\kappa=\sqrt{\frac{\bar{T}_{f}+\Delta L}{T_{n}}}
$$

Notice that $\kappa$ is not related to $L_{n}$. It can be related to the assumed error in $L_{n}$ trough $\Delta L$. The controller parameters $T_{i}$ and $K$ respond as in a normal PI controller. 
Table 1 FOPDT model parameters as well as $A$ and $\kappa$

\begin{tabular}{cccccc}
\hline$i$ & $T_{n}$ & $L_{n}$ & $\bar{T}_{f}$ & $T_{r}$ & $\kappa$ \\
\hline 1 & 10.4 & 6.8 & 0.27 & 1.7 & 6.2 \\
2 & 2.0 & 6.2 & 0.27 & 0.7 & 2.7 \\
3 & 3.0 & 9.1 & 1.06 & 1.8 & 1.7 \\
4 & 1.7 & 8.4 & 1.18 & 1.4 & 1.2 \\
5 & 0.8 & 5.4 & 0.17 & 0.4 & 2.4 \\
6 & 7.6 & 2.6 & 1.32 & 3.2 & 2.4 \\
7 & 1.3 & 5.7 & 0.17 & 0.5 & 2.7
\end{tabular}

\subsection{Simulation examples}

Simulation results for the PPI and the FPPI have been presented in [Hägglund, 1996; Normey-Rico et al., 1997; Ingimundarson and Hägglund, 2000b]. To give an idea of how conservative the design method presented is, a FPPI controller was designed for a collection of processes. They are shown here without the dead-time.

$$
\begin{array}{ll}
P_{1}(s)=\frac{1}{(10 s+1)(2 s+1)} & P_{2}(s)=\frac{1}{(s+1)^{3}} \\
P_{3}(s)=\frac{-s+1}{(s+1)^{5}} & P_{4}(s)=\frac{-2 s+1}{(s+1)^{3}} \\
P_{5}(s)=\frac{9}{(s+1)\left(s^{2}+2 s+9\right)} & P_{6}=\frac{0.5}{(s+1)}+\frac{0.05}{s+0.1} \\
P_{7}(s)=\frac{64}{(s+1)(s+2)(s+4)(s+8)} &
\end{array}
$$

The dead-time was always equal to 5 making the total process equal to

$$
P(s)=P_{i}(s) e^{-5 s}
$$

for $i$ ranging from 1 to 7 .

The results can be seen in Table 1 Notice that $T_{r}$ is in all cases smaller than $T_{n}$ which means that the closed-loop system has a faster step set point response than the open-loop one. Notice that the two processes with smallest $T_{n} / T_{r}$ ratio are non minimum phase and not monotonically decreasing. Since the response goes in the wrong direction in the beginning the area $A$ becomes quite large in those cases. This reduces the bandwidth through $\bar{T}_{r}$.

\subsection{Application to a tank lab process}

The above mentioned methods were applied to a tank laboratory process at the Department of Automatic Control in Lund. It consists of a tank with free outflow and a level sensor as well as a pump. The process has a long dead-time because the pump pumps the water into an open channel with a small slope. Obvious nonlinearities in the process are the relation of outflow to the height in the tank as well as non-symmetry because the pump cannot remove water from the tank. A PI controller was tuned and a closed-loop experiment performed followed by a an open-loop step experiment. The result of the open-loop experiment as well as the corresponding 


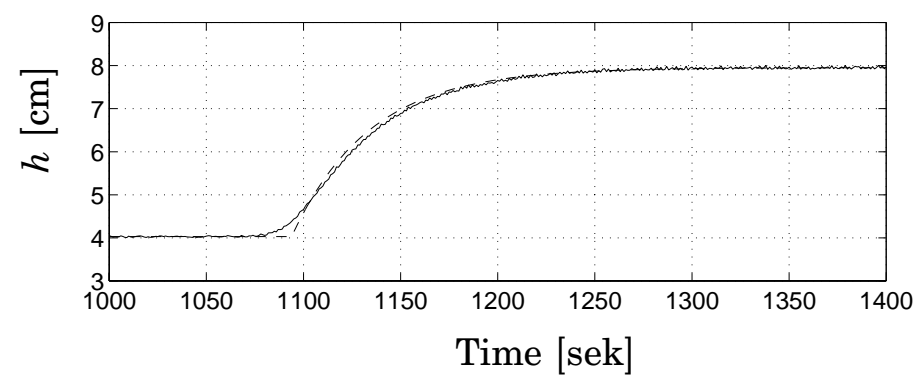

Figure 8 Open loop step response of the real tank (solid line), and the model (dashed line).
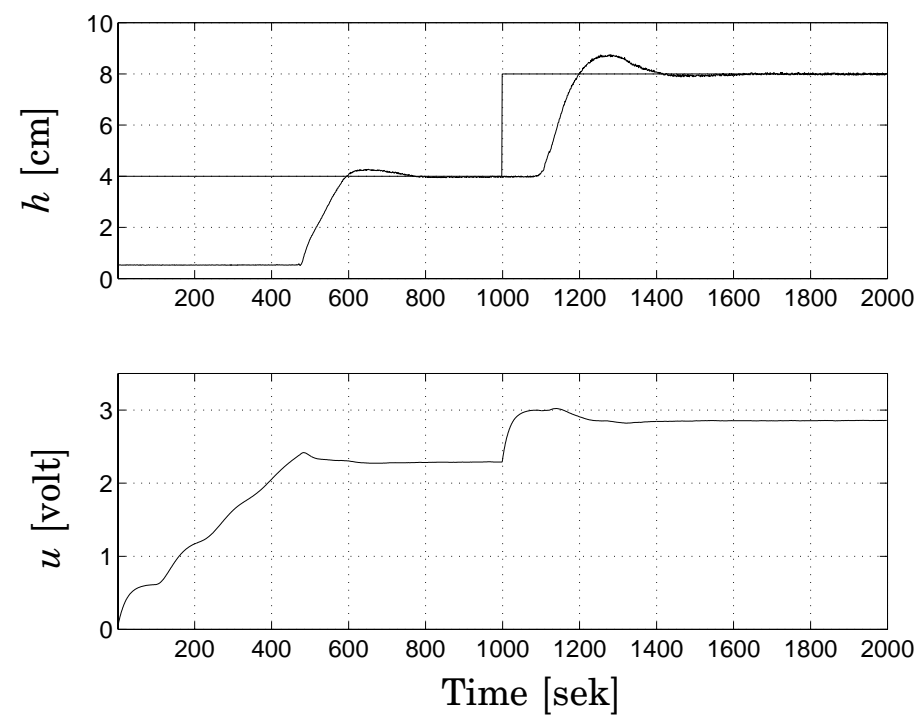

Figure 9 Closed loop step response of the real tank with a FPPI controller.

FOPDT response are shown in Fig. 8. The sampling time was 1 second. The identified FOPDT model was

$$
P_{n}(s)=\frac{5.6}{40.2 s+1} e^{-93.9 s}
$$

From the area between the responses $\bar{T}_{f}$ was found to be equal to 4.4 . This gave $T_{r}=13.3$. In Fig. 9 a set point step response can be seen. The gain in the proportional part of the PI controller was set to 0 from the set point to achieve a smoother response. The controller was started with a reference value of $4 \mathrm{~cm}$. Then an additional step was applied at time 1000 taking the level to $8 \mathrm{~cm}$. The over shoot is caused by unexplained nonlinearities. Reducing $\kappa$ considerably did not eliminate it. Otherwise a smooth control is observed.

\section{Unstable case, integrating processes.}

The extension of the Smith predictor to the unstable plant case was first presented in [Watanabe and Ito, 1981]. The compensator used here is the 


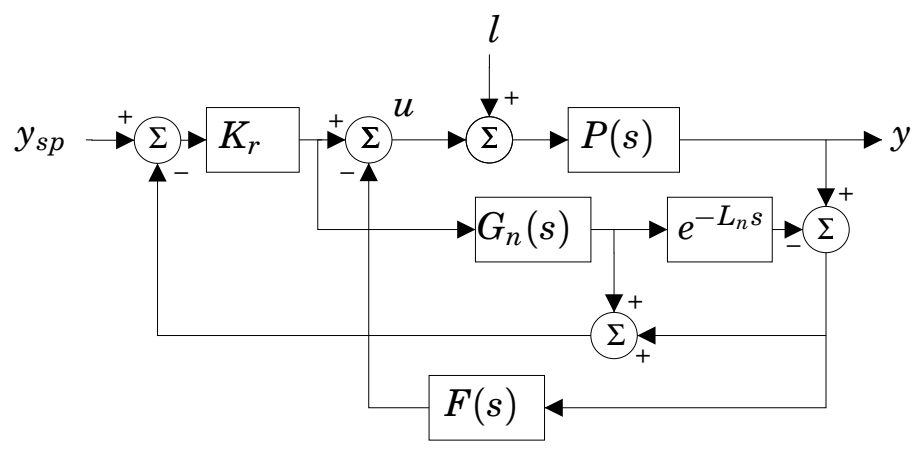

Figure 10 The improved dead-time compensating controller for integrating processes.

Modified Smith Predictor (MSP) presented in [Matausek and Micic, 1996]. There, care was taken to make sure all parameters could be related to identified parameters of the process model or to classical control theory concepts.

The block diagram of the dead-time compensator can be seen in Fig. 10. The model of the plant is the simple two-parameter model

$$
P_{n}(s)=G_{n}(s) e^{-L_{n} s}=\frac{K_{n}}{s} e^{-L_{n} s}
$$

The transfer function $F(s)$ is a constant $K_{0}$ which is related to the twoparameter model parameters as

$$
K_{0}=\frac{1}{2 L_{n} K_{n}}
$$

Introducing

$$
T_{r}=\frac{1}{K_{n} K_{r}}
$$

the transfer function from set point value $r(t)$ is in the nominal case given by

$$
\frac{Y(s)}{Y_{s p}(s)}=\frac{1}{T_{r} s+1} e^{-L_{n} s}
$$

$T_{r}$ has therefore the nice interpretation of being the time constant from set point to the output signal.

From the above equations it is clear that given a process model $P_{n}(s)$ the only parameter left to determine is $T_{r}$. That is the subject of the next section.

\subsection{Determining $T_{r}$}

In [Normey-Rico and Camacho, 1999] a DTC for integrating processes was proposed whose closed-loop time constant was related to asymptotes of the uncertainty norm-bound of the DTC. The same approach is taken here.

The error between the plant and the model is

$$
\delta P(s)=P(s)-P_{n}(s)
$$


The uncertainty norm-bound for the MSP or the maximum value of $|\delta P|$ allowed while keeping closed loop stability is

$$
|\Delta P|_{\mathrm{MSP}}=\frac{\left|K_{n}\left(j \omega T_{r}+1\right)\left(2 L_{n} j \omega+e^{-j L_{n} \omega}\right)\right|}{\left.\mid-\left(2 L_{n}+T_{r}\right) \omega^{2}+j \omega\right) \mid}
$$

This equation is obtained from the characteristic equation in a similar way as Eq. (17).

As pointed out in [Normey-Rico and Camacho, 1999] this bound depends almost entirely on $T_{r}$. The bound is shown in Fig. 11. For low frequencies the bound behaves as

$$
|\Delta P(i \omega)| \approx \frac{K_{n}}{\omega}
$$

For high frequencies the bound has an asymptote given by

$$
\lim _{\omega \rightarrow \infty}=\frac{2 K_{n} L_{n} T_{r}}{2 L_{n}+T_{r}}
$$

To evaluate the minimum of the uncertainty norm-bound it is fruitful to consider it a product of two factors, one of which is monotonically decreasing. The one that has local minima is

$$
f(\omega)=\left|\frac{2 j L_{n} \omega+e^{-j L_{n} \omega}}{\omega}\right|
$$

The minimum value of this function can be approximated with $L_{n}$. This gives a lower bound of $|\Delta P(i \omega)|$

$$
\beta=\frac{K_{n} L_{n} T_{r}}{2 L_{n}+T_{r}} \leq \min _{\omega}|\Delta P(i \omega)|
$$

Taking this into account when solving for $T_{r}$ gives

$$
T_{r}=\frac{2 L_{n} \beta}{K_{n} L_{n}-\beta}
$$

From closer inspection of Fig. 11 it can be concluded that for $L_{n}$ larger than 1, the bound given by Eq. (42) is conservative, and that the real minimum is much closer to the value given by Eq. (41).

To evaluate the value of $|\delta P(i \omega)|$ for each frequency, the assumption is made that the real process is given by a first-order plus dead-time transfer function with an integrator

$$
P(s)=\frac{K_{p}}{s(T s+1)} e^{-L s}
$$

The absolute value of $\delta P(i \omega)$ is then

$$
|\delta P(i \omega)|=\left|\frac{K_{p} e^{-i L \omega}-K_{n}(i T \omega+1) e^{-i L_{n} \omega}}{(i T \omega+1) i \omega}\right|
$$




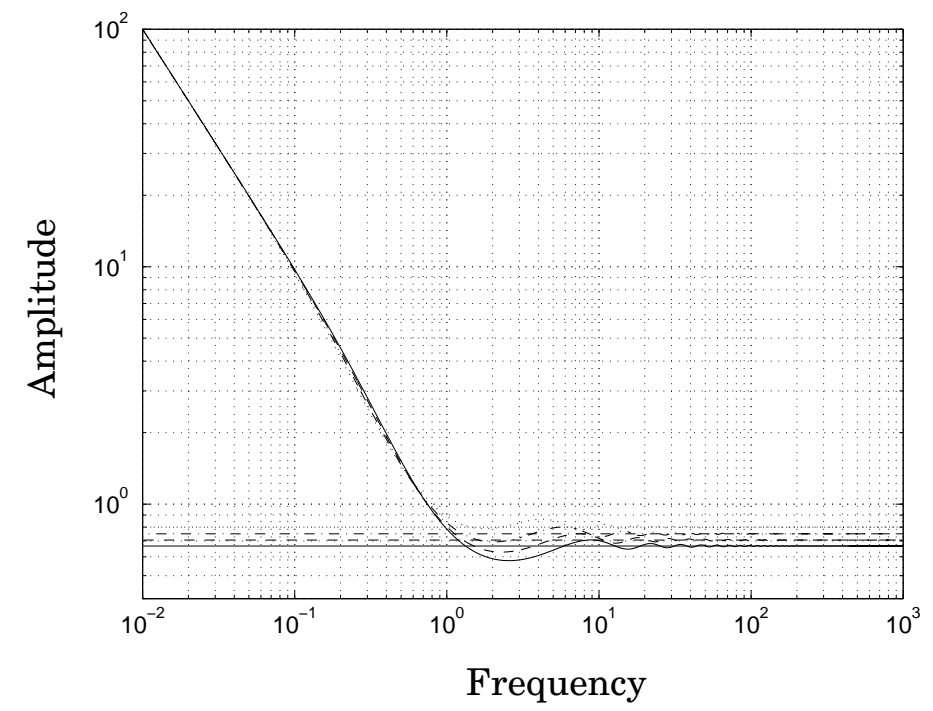

Figure 11 Uncertainty norm boundary for $T_{r}=K_{n}=1$ for varying $L_{n}$ equal to $1(-), 3(--), 5(-\cdot-)$ and $10(\cdots)$.

As pointed out in [Normey-Rico and Camacho, 1999] this function can be characterized by three main frequency intervals. For small $\omega$ this function behaves as

$$
|\delta P(i \omega)| \approx\left|\frac{K_{p}-K_{n}}{\omega}\right|
$$

For large $\omega$ this function has a slope of $20 \mathrm{~dB} / \mathrm{dec}$. For sufficiently high and low frequencies $|\delta P|$ will be smaller than $|\Delta P|$. It is the error at intermediate frequencies that is of most interest. This will be referred to as $\delta P_{0}$ in the following.

In [Normey-Rico and Camacho, 1999] the value at intermediate frequencies was approximately calculated by assuming that the velocity gains of the model and process were equal, $K_{p}=K_{n}$. Then $\delta P_{0}$ could be estimated as

$$
\delta P_{0}=\lim _{s \rightarrow 0} \delta P(s)=K_{n}\left(L+T-L_{n}\right)
$$

In [Normey-Rico and Camacho, 1999] $\delta P_{0}$ is viewed as a tuning parameter from which the closed-loop time constant is calculated. The methodology is therefore to assume an error between $L+T$ and $L_{n}$ and from there get the initial tuning.

Here the approach is to perform a simple open-loop experiment from where a upper bound is found on $\delta P_{0}$. The area between the actual response and the response of the model is calculated when the input is an impulse of height $h_{p}$ and duration $\tau_{p}$. Denoting as before $f(t)=y(t)-y_{n}(t)$ the following equation is the definition of the Laplace transform.

$$
\delta P(s) \frac{h_{p}\left(1-e^{-\tau_{p} s}\right)}{s}=\int_{0}^{\infty} e^{-s t} f(t) d t
$$

From this equation, by replacing the argument $s$ with $i \omega$ the following 


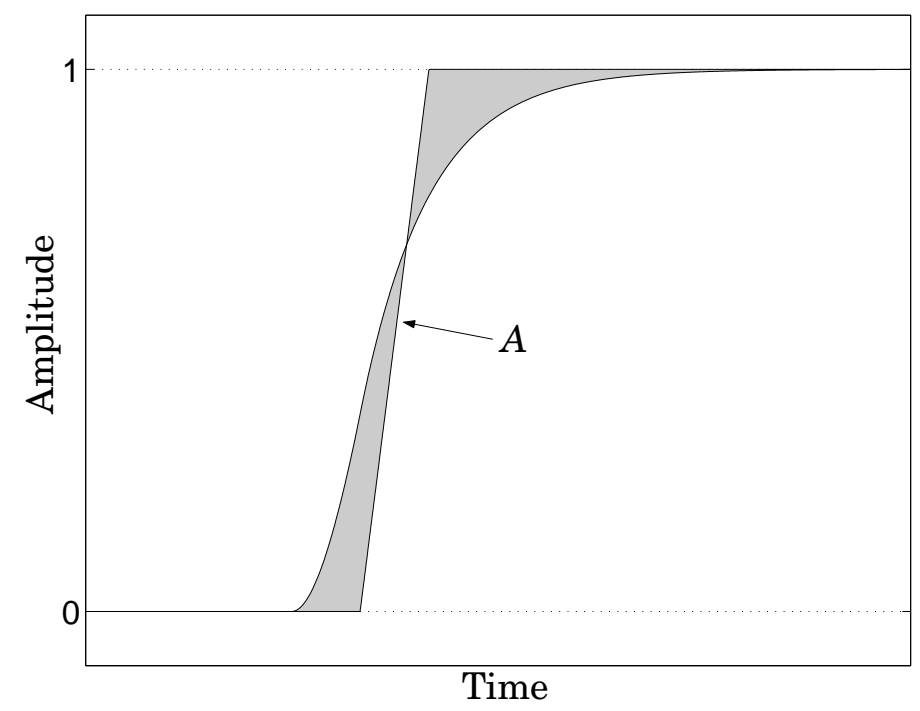

Figure 12 The integrating area.

inequalities are obtained.

$$
\begin{aligned}
\left|\delta P(i \omega) \frac{h_{p}\left(1-e^{-\tau_{p} i \omega}\right)}{i \omega}\right| & =\left|\int_{0}^{\infty} e^{-i \omega t} f(t) d t\right| \\
& \leq \int_{0}^{\infty}|f(t)| d t
\end{aligned}
$$

Notice that an upper bound on $\delta P_{0}$ can be obtained as

$$
\delta P_{0} \leq \frac{\int_{0}^{\infty}|f(t)| d t}{h_{p} \tau_{p}}=A
$$

The area $A$ has a nice graphical interpretation if the impulse response of the model and real process is normalized to 1 and plotted on the same graph. $A$ is then the area between the curves. This is shown in Fig. 12.

Notice that this bound on $\delta P_{0}$ is always larger and therefore more conservative than the value obtained by Eq. (44). Since $\delta P(i \omega)$ is weighted with $\left(1-e^{-i \tau_{p} \omega}\right) / \omega$ in the inequality, $\delta P(i \omega)$ can be arbitrary large when $\tau_{p} \omega=2 \pi$. The above method therefore does not guarantee stability but should work well for well-behaving processes. The gain is that it eliminates the need to tune the last parameter. Substituting $\beta$ with $A$ in Eq. (43) gives then the time constant $T_{r}$.

\subsection{Sensitivity to dead-time errors}

Given an error $\Delta L$ in the dead-time of the real process, the increase in integral $A$ would be maximum $\Delta L K_{n}$. Suspected variations in the deadtime can therefore be taken into account by increasing $\beta$ in Eq. (43) by $\Delta L K_{n}$.

If it is assumed that the process has the same structure as the model but a different dead-time, $L=L_{n}+\delta L$ the loop gain of the MSP is

$$
L(s)=\frac{1}{2} \frac{e^{-L s}\left(\left(2 L_{n}+T_{r}\right) s+1\right)}{s L_{n}\left(T_{r} s+1-e^{-L_{n} s}\right)}
$$




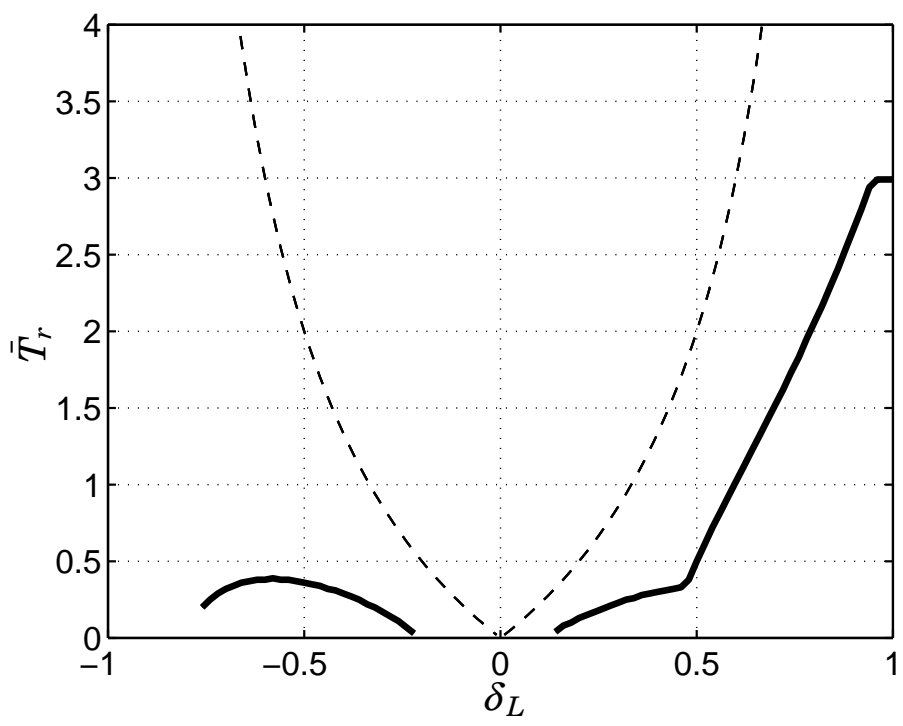

Figure 13 Thick line is the lower bound on $\bar{T}_{r}$ for a given error $\delta_{L}$. The dashed line is the design recommendation for $\bar{T}_{r}$ according to Eq. (43).

Using the same approach as in the previous section the following dimensionless variables are defined

$$
\delta_{L}=\Delta L / L_{n} \quad \bar{\omega}=\omega L_{n} \quad \bar{T}_{r}=T_{r} / L_{n}
$$

This gives the following dimensionless loop gain

$$
\bar{L}(i \bar{\omega})=\frac{1}{2} \frac{e^{-i\left(1+\delta_{L}\right) \bar{\omega}}\left(i\left(2+\bar{T}_{r}\right) \bar{\omega}+1\right)}{i \bar{\omega}\left(i \bar{T}_{r} \bar{\omega}+1-e^{i \bar{\omega}}\right)}
$$

Fig. 13 shows the lower bound on $\bar{T}_{r}$ to maintain stability as a function of $\delta_{L}$. The bound is not symmetric around 0 . Rather it is shown that an increase in dead-time is more likely to cause instability than a decrease. If $\bar{T}_{r}$ is larger than 0.4 it will be stable for any decrease in $\delta_{L}$ down to -1 . For an initial tuning which would give $\bar{T}_{r}$ equal to 0.01 , the controller would be stable for $\delta_{L} \in[-0.22,0.14]$.

The design rule given by Eq.(43) can be rewritten relating $\bar{T}_{r}$ to an assumed error $\Delta L$ by using $\beta=\Delta L K_{n}$. This results in

$$
\bar{T}_{r}=\frac{2 \delta_{L}}{1-\delta_{L}}
$$

This function is also shown in Fig. 13. The suggested $\bar{T}_{r}$ is well above the stability limit.

\subsection{Simulation examples}

To get an idea about what closed-loop time constant one can expect to obtain with the presented methodology, a two-parameter model was found for a collection of processes. The dynamics additional to the integrator and 
Table 2 Identification and tuning results for integrating processes.

\begin{tabular}{ccccc}
\hline$i$ & $T_{n}$ & $T_{r}$ & $A$ & $L_{n}$ \\
\hline 1 & 1 & 0.8 & 0.4 & 6.0 \\
2 & 1.0 & 0.8 & 0.4 & 6.1 \\
3 & 2.0 & 2.1 & 0.9 & 8.0 \\
4 & 3.0 & 2.8 & 1.2 & 11.0 \\
5 & 1.7 & 3.2 & 1.4 & 10.0 \\
6 & 0.8 & 0.7 & 0.3 & 6.2 \\
7 & 7.6 & 21.5 & 5.3 & 10.6 \\
8 & 1.3 & 1.1 & 0.5 & 6.9 \\
\hline
\end{tabular}

dead-time are shown below as $P_{1}(s)-P_{8}(s)$

$$
\begin{array}{ll}
P_{1}=\frac{1}{s+1} & P_{2}=\frac{1}{(0.1 s+1)(s+1)} \\
P_{3}=\frac{1}{(s+1)^{3}} & P_{4}=\frac{-s+1}{(s+1)^{5}} \\
P_{5}=\frac{-2 s+1}{(s+1)^{3}} & P_{6}=\frac{1}{(s+1)\left(s^{2}+2 s+9\right)} \\
P_{7}=\frac{0.5}{(s+1)}+\frac{0.05}{(s+0.1)} & \\
P_{8}=\frac{64}{(s+1)(s+2)(s+4)(s+8)} &
\end{array}
$$

The dead-time, $L$, was equal to 5 in all simulations. The total process was therefore

$$
P(s)=P_{i}(s) \frac{1}{s} e^{-5 s}
$$

The resulting $T_{r}$ is shown in Table. 2. In [Matausek and Micic, 1996] it was suggested that a suitable value of $T_{r}$, given that this value would be available, could be the apparent time constant of the dynamics additional to the integrator or $P_{i}(s) e^{-5 s}$. This is shown as well in the table as $T_{n}$. Notice that $L_{n}$ is the dead time of the two-parameter model. Comparing $T_{r}$ and $T_{n}$ one can see that usually they are quite close. Often $T_{r}$ is a little bit smaller than $T_{n}$. Exceptions to this are processes 5 and 7. Process 5 is non-minimum phase while process 7 has a slow zero giving a large area $A$ compared to $T_{n}$. In the case of process $7, A$ is also very large compared to $L_{n}$. This results in a large $T_{r}$ according to Eq. (43).

Closed-loop set point and load disturbance responses are shown in Figs. 14 to 16 for a selection of processes when $T_{r}$ is found from Eq. (43). Also shown (dashed line) are simulations when $T_{r}$ is set equal to $T_{n}$.

\subsection{Practical issues}

Some remarks on the practical use of the methodology presented are in order. Most of the remarks made in Section. 4.3 apply here as well. Notice that when $A$ is close to $K_{n} L_{n}, T_{r}$ becomes very large calculated with Eq.(43).

\subsection{Extensions}

In [Matausek and Micic, 1999] an extension to the MSP was proposed. To improve load disturbance rejection the transfer function $F(s)$ should have 

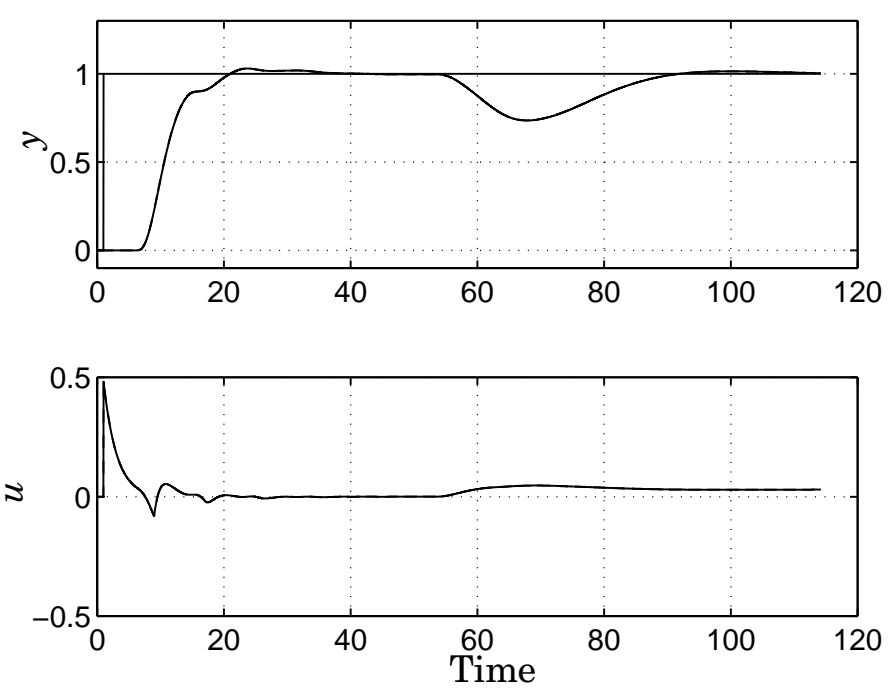

Figure 14 Simulation example. Process 3.
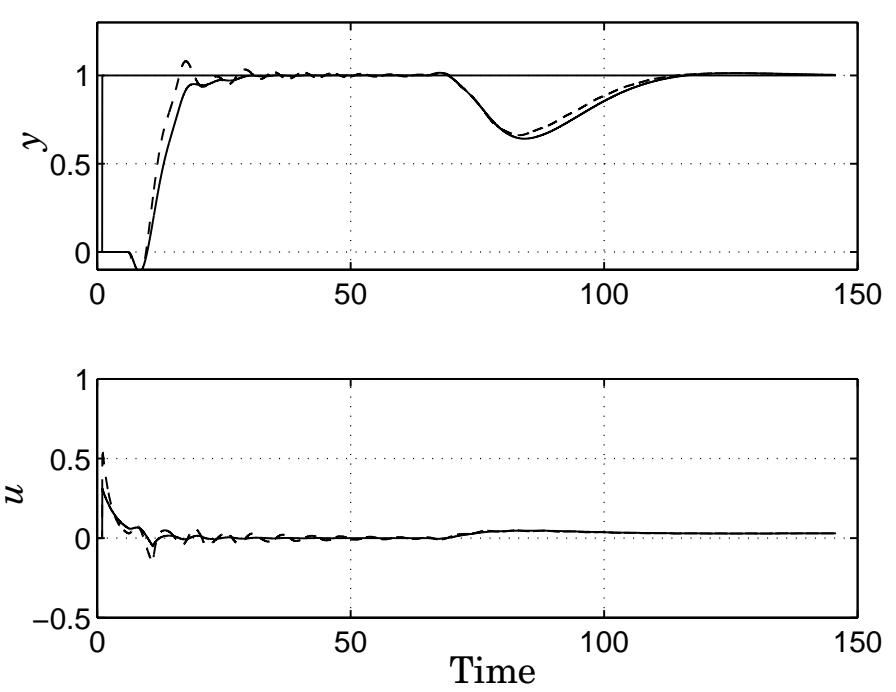

Figure 15 Simulation example. Process 5.

the form

$$
F(s)=K_{0} \frac{s T_{d}+1}{s T_{d} / 10+1}
$$

where $T_{d}=0.4 L_{n}$ and $K_{0}=0.6 /\left(L_{n} K_{n}\right)$. The form of the transfer function is similar to a PD controller. It's purpose is also to predict the load disturbances $l$ better which in turn leads to better disturbance rejection. Simulation experience indicates it is possible to use this extension with the tuning procedure presented. Sometimes the increase in conservativeness associated with the procedure is welcomed. In Fig. 17 the Nyquist diagram is shown for process 5 when $T_{r}$ is found by the procedure presented and as recommended in [Matausek and Micic, 1996]. It can be seen that the latter results in an unstable closed-loop system. 

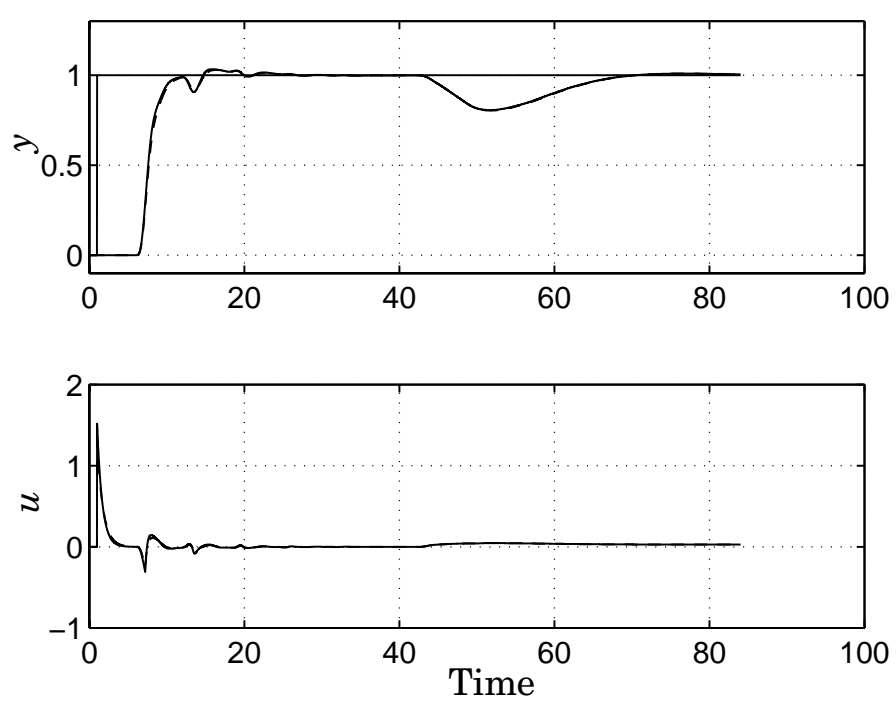

Figure 16 Simulation example. Process 6.

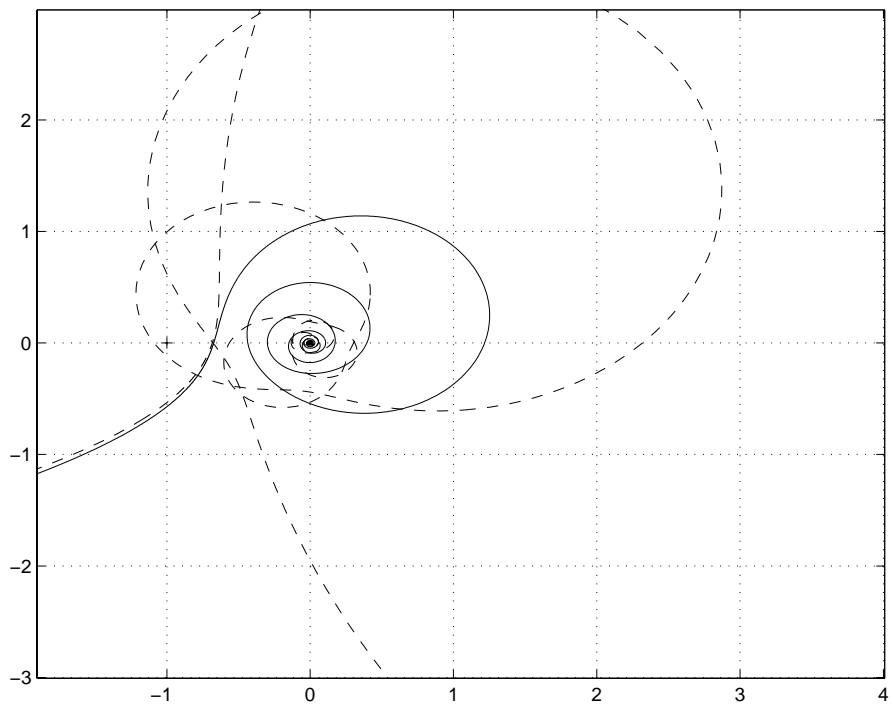

Figure 17 Nyquist curve for process $5, L=10$. Extended MSP with $T_{r}$ found from Eq.(43), (-). Extended MSP with $T_{r}$ set equal to apparent time constant, $T_{r}=1.7,(--)$.

\subsection{Application to a lab process}

The method presented was used on the tank laboratory process presented in Section 4 after some modification of the process. To make an integrating process a tank without an outflow hole was added under the first one. The controlled variable was therefore the height in the second tank. To avoid nonlinearities at low flow levels, a second pump was installed which pumped with a constant flow rate out of the lower tank. In this way the first pump was set to work around a constant flow rate corresponding to 3 Volts.

The identification experiment was performed manually because it was 

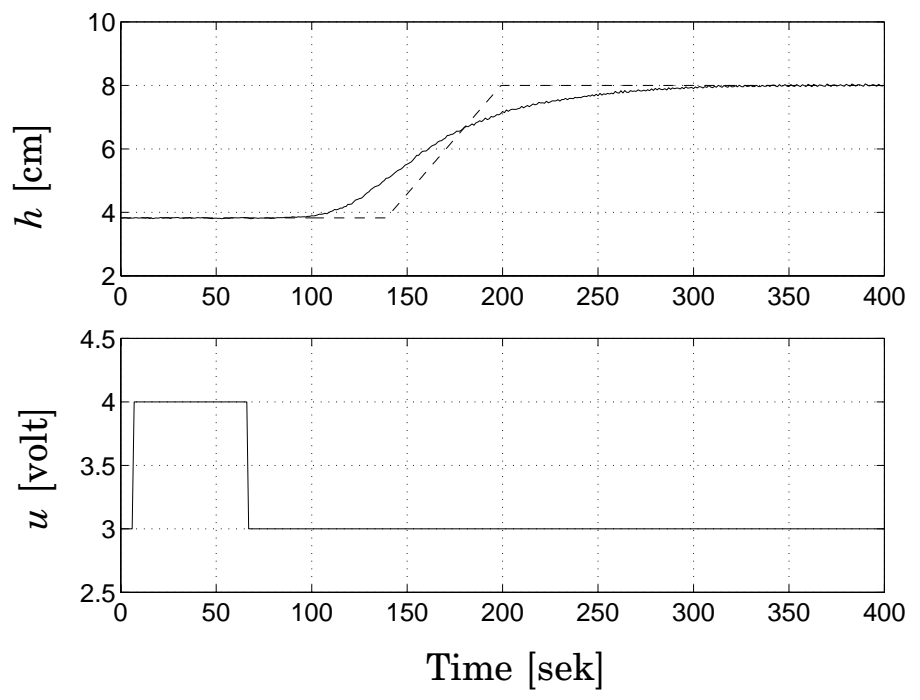

Figure 18 Identification experiment for integrating case. The upper graph shows the impulse response of real tank $(-)$ and that of the two-parameter model (--).

difficult to tune a suitable PI controller. An impulse of height 1 Volt and duration 60 seconds was added to the equilibrium value of 3 Volts. The response of the tank and the identified two-parameter model are shown in Fig. 18. The identified two-parameter model was

$$
P_{n}(s)=\frac{0.07}{s} e^{-132.5 s}
$$

The area $A$ between the responses was 1.6. This resulted in a closed-loop time constant $T_{r}=54.8$ seconds. In Fig. 19 a closed-loop step response experiment using the MSP is shown. The controller was started at time 1000 while a step was applied at time 2000 taking the level in the tank from $4 \mathrm{~cm}$ to $8 \mathrm{~cm}$. The controller performs as expected. The step response looks similar to what was seen in simulation.

\section{Conclusions}

In this paper tuning procedures for dead-time compensators have been presented. Dead-time compensators for both stable and integrating processes are considered. The closed-loop time constant is found by comparing the model output to the process output when a simple open-loop experiment is performed.

In the case of integrating processes the procedure eliminates the need to manually tuning one parameter.

The DTC's are simple and can be manually fine-tuned or re-tuned. It is also shown how the DTC's can be given a guaranteed delay-margin.

Finally the methodology has been applied to a laboratory process with success.

Åström, K. J. and T. Hägglund (1995): PID Controllers: Theory, Design, and Tuning. Instrument Society of America, Research Triangle Park, North Carolina. 

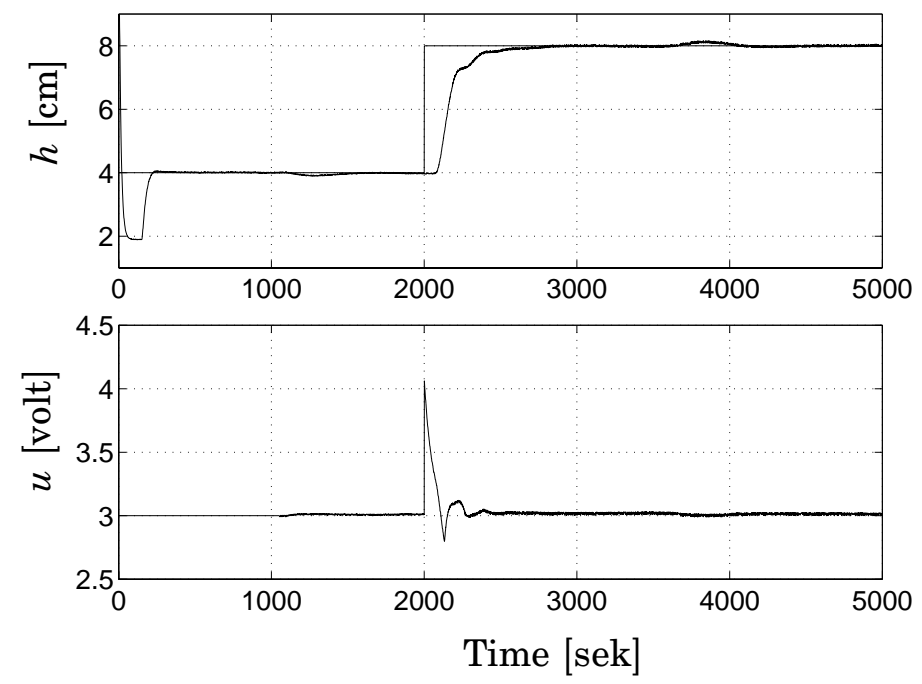

Figure 19 Step response of the real tank.

Hägglund, T. (1996): "An industrial dead-time compensating PI controller." Control Engineering Practice, 4, pp. 749-756.

Ingimundarson, A. and T. Hägglund (2000a): "Closed-loop identification of first-order plus dead-time model with method of moments." In ADCHEM 2000, IFAC International Symposium on Advanced Control of Chemical Processes. Pisa, Italy.

Ingimundarson, A. and T. Hägglund (2000b): "Robust automatic tuning of an industrial PI controller for dead-time systems." In IFAC Workshop on Digital Control - Past, present, and future of PID Control. Terrassa, Spain.

Landau, I., D. Rey, A. Karimi, A. Voda, and A. Franco (1995): "A flexible transmission system as a benchmark for robust digital control." Proceedings of the Third European Control Conference, Rome, Italy.

Lee, T., Q. Wang, and K. Tan (1995): "Automatic tuning of the smith predictor controller." Journal of Systems Engineering, $\mathbf{5}$.

Lee, T., Q. Wang, and K. Tan (1996): "Robust smith-predictor controller for uncertain delay systems." AIChE Journal, 42.

Matausek, M. and A. Micic (1996): "A modified smith predictor for controlling a process with an integrator and long dead-time." IEEE Transaction on Automatic Control, 41.

Matausek, M. and A. Micic (1999): "On the modified smith predictor for controlling a process with an integrator and long dead-time." IEEE Transaction on Automatic Control, 44.

Morari, M. and E. Zafiriou (1989): Robust Process Control. Prentice-Hall, Englewood Cliffs, New Jersey.

Normey-Rico, J., C. Bordons, and E. Camacho (1997): "Improving the robustness of dead-time compensating pi controllers." Control Engineering Practice, 5, pp. 801-810. 
Normey-Rico, J. and E. Camacho (1999): "Robust tuning of dead-time compensators for processes with an integrator and long dead-time." IEEE Transaction on Automatic Control, 44.

Palmor, Z. (1980): "Stability properties of smith dead-time compensator controllers.” Int. J. Control, 32.

Palmor, Z. and M. Blau (1994): "An auto-tuner for smith dead time compensator." Int. J. Control, 60.

Smith, O. J. M. (1957): "Closed control of loops with dead time." Chemical Engineering Progress, 53, May, pp. 217-219.

Vrancić, D., D. Vrecko, D. Juricić, and S. Strmcnik (1999): "Automatic tuning of the flexible smith predictor controller." Proceedings of the American Control Conference, San Diego, California.

Watanabe, K. and M. Ito (1981): "A process-model control for linear systems with delay.” IEEE Transaction on Automatic Control, 26. 